\title{
Isolierung von gelösten organischen Verbindungen aus dem Meerwasser unter besonderer Berücksichtigung der Aminosäuren
}

\author{
HEINZ SCHAEFER ${ }^{1}$ \\ Biologische Anstalt Helgoland, Meeresstation, Helgoland
}

\begin{abstract}
Isolation of dissolved organic substances from sea water with special reference to amino acids. Several methods have been used hitherto to isolate amino acids from sea water. These methods are discussed in the first part of this paper, which is primarily concerned with the presentation of a new method, the desalting efficiency of which had been proved in a previous work by the author. In the newly described procedure, principal removal of inorganic salts is accomplished by ion retardation using resin Retardion 11 A8 (Dow Chemical Company, Midland, Mich., USA). The remaining ions and most of the organic compounds are adsorbed by ion exchange columns. It is thus possible to separate amino acids, carbohydrates and organic acids and obtain them in a salt-free solution. 'The new procedure very efficiently isolates some main groups of organic substances from one liter of sea water. Single components of these groups are then identified by paper chromatography. Employing the new method, several amino acids, carbohydrates, ascorbic acid and two organic acids could be isolated from sea water samples of the Deutsche Bucht (Southern North Sea) and from plankton cultures.
\end{abstract}

\section{EINLEITUNG}

Die Gesamtmenge der gelösten organischen Substanzen des Meerwassers wird normalerweise als "gelöster organischer Kohlenstoff" bestimmt. Eine Einteilung in einzelne Komponenten, die mehr oder weniger definierten Substanzgruppen zugehören, wird zuweilen versucht. In diesen Fällen wird die organische Substanz zum Beispiel als organischer N oder P oder als Gesamtkohlenhydrate angegeben. Jedoch geht aus diesen Untersuchungen häufig nicht hervor, welche Substanzen im einzelnen auftreten und wie groß der Anteil an biologisch verwertbaren Stoffen ist. Dies ist eine Frage von eminenter Bedeutung nicht nur für die Kenntnis der Stoffkreislaufes im Meer, sondern auch für das Verständnis der Okologie und des Verhaltens der marinen Lebewesen. Die summarische C-Analyse bringt uns hier keineswegs weiter. Wir müssen wissen, um welche Verbindungen es sich im einzelnen handelt und in welchen Konzentrationen diese vorkommen.

Die Durchführung derartiger Analysen ist erst in den letzten Jahren seit der Aus-

\footnotetext{
1 Der Deutschen Forschungsgemeinschaft danke ich für die Unterstützung der Arbeit. $43-45$.

Neue Anschrift des Verfassers: Dr. H. Schaefer, 673 Neustadt/Weinstr., Maximilianstr.
} 
arbeitung verfeinerter mikrochemischer Untersuchungsverfahren möglich geworden. Aber auch heute ist noch eine große Schwierigkeit zu überwinden. Die einzelnen Substanzen kommen nämlich in außerordentlich geringen Mengen vor - im günstigsten Fall in Konzentrationen von etwa $1 \mathrm{mg} / \mathrm{l}$ Meerwasser. Vor der qualitativen und quantitativen Analyse steht daher die Abtrennung der organischen Stoffe von der vergleichsweise riesigen Menge anorganischer Salze. Bisher sind für diese Abtrennung verschiedene Methoden vorgeschlagen worden (vgl. Johnston 1955, Jefrrey \& Hood 1958), die sich für Substanzgruppen oder einzelne Substanzen mehr oder weniger bewährt haben. Ein Universalverfahren zur Isolierung sämtlicher organischer Verbindungen aus dem Meerwasser existiert jedoch nicht. Es muß daher zunächst versucht werden, eine Substanzgruppe nach der anderen zu bearbeiten und leistungsfähige Methoden für ihre Isolierung zu finden.

Eine der wichtigsten Gruppen ist die der Aminosäuren. Letztere besitzen eine mannigfaltige Bedeutung im Stoffwechselgeschehen und lassen sich heute durch sehr gute Mikroverfahren nachweisen. Das ist wohl auch der Grund, daß auf diesem Sektor der organischen Meereschemie bisher am meisten gearbeitet worden ist. Auch die vorliegende Abhandlung befaßt sich vor allem mit der Isolierung der Aminosäuren und verwandter Verbindungen aus dem Meerwasser. Im Anschluß an eine Diskussion der Arbeiten anderer Autoren (auf mikrobiologische Methoden wird nicht eingegangen; vgl. dazu BeLser 1959) wird ein neues Isolierungsverfahren beschrieben. Ein kurzer Abschnitt ist der Isolierung von Ascorbinsäure und von organischen Säuren vorbehalten, deren Auffindung mit der neuen Methode in einem gewissen Maße gleichfalls möglich ist. Ferner wird kurz auf die Kohlenhydrate eingegangen, die auf die geschilderte Weise sehr gut isoliert werden können.

$\mathrm{Da}$ die eigenen Arbeiten infolge meiner Übersiedlung nach Neustadt/Weinstraße abgebrochen werden mußten, konnten die Anwendungsbeispiele nicht mehr quantitativ ausgewertet werden. Soweit es möglich ist, werden jedoch die Größenordnungen der Konzentrationen einiger gefundener Aminosäuren angegeben.

\section{METHODIK}

\section{Bisherige Isolierungsmethoden}

Eine Kritik mehrerer Isolierungsverfahren verfaßten JEFFrey \& Hood (1958). Dem Bearbeiter des Problems der organischen Substanzen im Meerwasser drängt sich zunächst die Isolierung mit Hilfe des Ionenaustauschverfahrens auf. Grundsätzlich ist diese Methode natürlich geeignet. Jedoch werden große Mengen an Austauschermaterial sowie an Elutions- und Regeneriermitteln benötigt. Angesichts des Vorkommens großer Mengen verschiedener anorganischer Ionen muß sehr kritisch geprüft werden, welches Elutionsmittel in Frage kommt. Bei Verwendung eines starken Kationenaustauschers halte ich in Übereinstimmung mit Palmork (1963a) eine Piperidinlösung zur Elution der Aminosäuren für sehr geeignet. Ich benutzte aber $0,3 \mathrm{~m}$ Piperidinlösung, um das Elutionsvolumen wesentlich zu verringern. Gegenüber der Elution mit Ammoniak oder verdünnter $\mathrm{HCl}$ bietet obige Lösung erhebliche Vorteile (BuchanaN 
1957). Nach Johnston (1955) und JeFFrey \& Hoov (1958) ist das Ionenaustauschverfahren dann günstig, wenn das Meerwasser durch andere Methoden vorentsalzt wird, so daß dann nur noch geringe Mengen Austauschermaterial in Frage kommen. So setzten auch Tatsumoto et al. (1961), Palmork (1963a) und Degens (1964) das Austauschverfahren nur zur Restentsalzung ein; dasselbe Prinzip erscheint auch in meiner eigenen Isolierungsmethode.

Nach JefFrey \& Hood (1958) soll ein großer Teil der organischen Stoffe durch Zusatz von $0,1 \mathrm{~m} \mathrm{FeCl}_{3}$ zum Seewasser quantitativ kopräzipitiert werden. Aus dem Präzipitat müssen dann die organischen Substanzen isoliert werden. Tatsumoto et al. fanden jedoch bei Anwendung dieser Methode nur noch $53 \%$ einer zugesetzten Aminosäure im Konzentrat wieder. Bei PARK et al. (1962) fehlen Hinweise auf die Ausbeute. In eigenen Versuchen mit dieser Methode wurden bestenfalls nur $40 \%$ von zugesetztem Glycin oder Glucose wiedergefunden. Die Eignung des Verfahrens dürfte fraglich sein.

Newell (1962) versuchte, organische Substanzen des Seewassers mit Aluminiumund Magnesiumhydroxiden zu fällen. Anorganische und organische Phosphate wurden quantitativ niedergeschlagen, ihre Konzentration konnte exakt ermittelt werden. Zur Identifizierung der organischen Phosphate müßten allerdings die niedergeschlagenen anorganischen Substanzen wieder entfernt werden. Für die Isolierung der Phosphate scheint das Verfahren vielversprechend zu sein. Eigene Versuche, auf diese Weise Aminosäuren aus dem Meerwasser abzutrennen, lieferten aber nur eine sehr geringe Ausbeute zugesetzter Testsubstanzen. Angewandt auf die Isolierung von Aminosäuren dürte für die NEwellsche Methode das gleiche gelten, was zur Kopräzipitationsmethode mit Eisensalzen gesagt wurde.

PALMORK (1963a) engte Seewasser im Rotationsverdampfer ein, wobei von Zeit zu Zeit die ausgefallenen Salze abfiltriert wurden. Das Restkonzentrat wurde durch eine Säule mit DOWEX 50 W geleitet und die adsorbierten Aminosäuren mit Piperidinlösung eluiert. Das Einengen des Seewassers muß also mehrmals unterbrochen werden, wodurch zusätzliche Arbeitsschritte erforderlich sind. Die ausgefällten Salze müssen sehr sorgfältig extrahiert werden, um mögliche Verluste $\mathrm{zu}$ vermeiden. Uber die Ausbeute an organischen Substanzen ist bei diesem Verfahren nichts angegeben worden.

Bei dem von JefFrey \& Hood (1958) diskutierten Dialysierverfahren wird ebenfalls stufenweise gearbeitet. Es wird so lange dialysiert, bis eine Salzkonzentration von $1 \%$ erreicht ist. Dann wird eingeengt bis zu einer Konzentration von 30 bis $40 \%$, weiter dialysiert etc., bis die Vollentsalzung eingetreten ist. Abgesehen von zahlreichen Arbeitsschritten dürfte dieses Verfahren wenig geeignet sein, weil die Ausbeute für manche zugesetzten Aminosäuren sehr gering ist.

PALMORK (1963b) isolierte die Aminosäuren als DNP-Derivate, die sich chromatographisch sehr gut nachweisen lassen. Ein Vorteil des Verfahrens ist, daß das Konzentrieren großer Flüssigkeitsmengen unterbleibt. Jedoch ist das Verfahren umständlich, über die Ausbeute zugesetzter Aminosäuren ist nichts bekannt.

Degens et al. (1964) dampften angesäuertes Meerwasser zur Trockne ein und extrahierten den Rückstand mehrmals mit 80\% \% Agem Athanol. Das alkoholische, noch sehr salzreiche Konzentrat wurde nach Abdampfen des Alkohols durch Ionenaustau- 
scher entsalzt und papierchromatographisch analysiert. Bei ausreichender Extraktion des Rückstandes sollen gute Ausbeuten erreicht worden sein. Eigene Versuche mit alkoholischer Extraktion von eingeengtem Seewasser führten zu unterschiedlichen Ergebnissen. Es wurden 72 bis $100 \%$ zugesetzten Glycins wiedergefunden. Im Extrakt war noch eine erhebliche Salzmenge verblieben, der Entsalzungsgrad, bezogen auf $\mathrm{Cl}^{7}$, betrug in diesen Versuchen etwa $11 \%$. Das aber bedeutet, daß eine sehr große Menge an Ionenaustauschern zur Restentsalzung erforderlich ist. Kritisch ist auch das Eindampfen des Meerwassers. Es muß sorgfältig darauf geachtet werden, daß Salze und damit ein Teil der organischen Substanzen nicht an den Glaswänden haften bleiben. Es empfiehlt sich daher, das Konzentrieren des Meerwassers nur so lange durchzuführen, bis noch einige $\mathrm{ml}$ Wasser übrig sind. Die Entfernung des restlichen Wassers erfolgt dann am besten durch Gefriertrocknung. Durch Verringerung des zur Extraktion benutzten Alkoholvolumens läßt sich der Entsalzungsgrad noch verbessern, jedoch muß auf eine erschöpfende Extraktion geachtet werden.

Schließlich dampte StEPHens $(1962,1963)$ Seewasser auf einer heißen Platte ein und extrahierte die Aminosäuren aus dem Rückstand mit $2 \% \mathrm{HCl}$ in Aceton. Der Extrakt wurde wieder eingedamptt und die mit Wasser gelösten Aminosäuren bestimmt. Die Ausbeute für einige getestete Aminosäuren betrug nur 54,5 bis 55,5\%.

\section{Eigene Untersuchungen: Die Retardion-Methode}

Zur Isolierung von Aminosäuren aus dem Meerwasser benutzte ich das Ionenverzögerungsverfahren mit Retardion 11A8. Uber die Entsalzung von salzreichen, organische Stoffe enthaltenden Lösungen mit Retardion habe ich früher ausführlich berichtet (ScHAEFFr 1964, 1965). Mit den dort angegebenen Säulenabmessungen konnten 50 bis $100 \mathrm{ml}$ Salzlösung weitgehend deionisiert werden, wobei der größte Teil der zugesetzten organischen Testsubstanzen, insbesondere Aminosäuren, Kohlenhydrate und Phosphorsäureester quantitativ wiedergefunden wurde.

Um 11 Meerwasser partiell entsalzen zu können, wurden nach eingehenden Vorversuchen 3 mit Retardion gefüllte Säulen folgender Abmessungen verwandt: (1) Säule $80 \times 6 \mathrm{~cm}$, Betthöhe Retardion $71 \mathrm{~cm}$, (2) Säule $80 \times 4 \mathrm{~cm}$, Betthöhe $67 \mathrm{~cm}$, (3) Säule $70 \times 2 \mathrm{~cm}$, Betthöhe $64 \mathrm{~cm}$. Die Vorversuche mit diesen Säulen waren die gleichen, wie sie in den erwähnten Arbeiten mit kleinen Säulen durchgeführt worden waren. Auf die erste Säule wurde mittels Schliffverbindung ein die Seewasserprobe enthaltender birnenförmiger Kolben aufgesetzt. Nach Durchlauf des Seewassers wurde die Säule an ein hochstehendes Wasserreservoir angeschlossen. Die einzelnen Säulen waren mit Kunststoffschläuchen von 1 bis $2 \mathrm{~mm}$ Durchmesser verbunden. Der Auslauf der letzten Säule konnte mit einem ähnlichen Schlauch entweder an ein Auffanggefäß oder an eine Ionenaustauschersäule angeschlossen werden. Die Durchführung der Entsalzung wurde in der früheren Arbeit (SCHAERER 1964) beschrieben. Vorversuche mit angeschlossenem Fraktionssammler (15 ml Fraktionen) ergaben, daß die Glycin oder Glucose enthaltende Fraktion einer 11 Meerwasserprobe die Säulen zwischen 1600 und $3100 \mathrm{ml}$ Auslauf einschließlich Meerwasserprobe verließ. Diese Fraktion wird im folgenden immer als "organische Fraktion " bezeichnet. Nach dem Durch- 
lauf der Wasserprobe wurde mit 12 bis 151 aqua destillata nachgespült, dann konnte die nächste Probe aufgegeben werden. Wie schon früher erläutert wurde, erfolgt die Elution der Retardionsäulen normalerweise mit aqua destillata.

Eine Reihe von Versuchen ergab, daß in der "organischen Fraktion" noch Restsalze enthalten waren. Thre Konzentration und der Entsalzungsgrad, also der Prozentsatz der entfernten Ionen, sind in Tabelle 1 wiedergegeben. Es ergibt sich daraus, daß

Tabelle 1

Durchschnittswerte von 4 Entsalzungsversuchen mit Retardion 11A8. Probe: 11 künstliches Meerwasser

\begin{tabular}{|cccc|}
\hline Ionen & vorgelegt & $\begin{array}{c}\text { in der ,organischen } \\
\text { Fraktion }{ }^{*} \text { verblieben }\end{array}$ & Entsalzungsgrad \\
\hline $\mathrm{Cl}^{-1}$ & $19,34 \mathrm{~g}$ & $2,715 \mathrm{~g}$ & $85,96 \%$ \\
$\mathrm{SO}_{4}^{-}$ & $2,72 \mathrm{~g}$ & $1,599 \mathrm{~g}$ & $41,22 \%$ \\
$\mathrm{Ca}^{++}$ & $416 \mathrm{mg}$ & $25,7 \mathrm{mg}$ & $93,82 \%$ \\
$\mathrm{Mg}^{++}$ & $1,295 \mathrm{~g}$ & $0,055 \mathrm{~g}$ & $95,76 \%$ \\
\hline
\end{tabular}

ein großer Teil der Ionen durch die Anwendung der Retardionsäulen in einem Arbeitsgang aus dem Meerwasser entfernt werden können. Weitere Vorversuche ergaben, daß zugesetztes Glycin im Eluat zwischen 90 bis $100 \%$ wiedergefunden wurde.

Zur exakten Analyse der im Meerwasser enthaltenen Aminosäuren durch Mikronachweisverfahren muß aber eine Vollentsalzung durchgeführt werden. $\mathrm{Zu}$ diesem Zweck wird die letzte Retardionsäule während des Auslaufes der „organischen Fraktion" (zwischen 1600 und $3100 \mathrm{ml}$ ) mittels Kapillarschlauch mit einer Austauschersäule von etwa $200 \mathrm{ml}$ Volumen verbunden. Dadurch werden die entsprechenden auslaufenden Ionen und die Aminosäuren adsorbiert. Nach Durchspülen dieser Säule mit 500 bis $1000 \mathrm{ml}$ aqua destillata werden die Aminosäuren eluiert. Bei Verwendung von Dowex $50\left(\mathrm{H}^{+}\right.$-Form $)$bewährte sich dabei, wie bereits eingangs erwähnt wurde, eine 0,3 m Piperidinlösung. Die Aminosäuren erschienen bei dieser Elutionsform im Bereich von etwa 750 bis $1000 \mathrm{ml}$, wobei für Routineuntersuchungen aus Sicherheitsgründen (zum Beispiel bei ungenügender Regeneration) ein Elutionsvolumen von 600 bis $1100 \mathrm{ml}$ gesammelt wurde. Diese salzfreie Fraktion wurde mehrmals im Rotationsverdampfer eingeengt und anschließend gefriergetrocknet.

Bei Verwendung des Anionenaustauschers Dowex 2 ( $\mathrm{OH}-$-Form) wurden die Aminosäuren mit n-Essigsäure eluiert. Anhand der Farbveränderung des Harzes ist der Durchlauf der Essigsäure leicht erkennbar. Kurz vor dem Durchbruch des Elutionsmittels wurde ein Auffanggefäß für die Aminosäurenfraktion angeschlossen und ein Volumen von etwa $500 \mathrm{ml}$ gesammelt, das ebenfalls zur Trockne gebracht wurde. Bei Verwendung des Anionenaustauschers ist jedoch zu beachten, daß einige Aminosäuren, besonders Arginin, nicht oder nur teilweise zurückgehalten werden.

Vorversuche ergaben, daß auf die Austauschersäulen aufgegebene Aminosäurelösungen, die eine Salzkonzentration wie die „organische Fraktion“ der Retardionsäulen enthielten, ebenfalls zwischen 95 bis $100 \%$ wiedergefunden wurden.

Aus praktischen Gründen empfiehlt es sich, folgende Zeiten bei diesem Voll- 
entsalzungsverfahren einzuhalten: Am frühen Morgen wird die Seewasserprobe bei etwa 400 bis 500 Torr durch ein Membranfilter (0,25 $\mu$ Porenweite) filtriert und sofort auf die Retardionsäulen gegeben. Bei stärkerer Trübung und größerem Planktongehalt erfolgt eine Vorfiltration durch ein engmaschiges Perlonfilter. Die Durchflußgeschwindigkeit wird durch eine Schraublklemme so geregelt, daß bis zum Abend 11 Seewasser und $600 \mathrm{ml}$ aqua destillata durch die Säulen gelaufen sind. Anschließend wird die Austauschersäule angeschlossen und wiederum die Durchlaufgeschwindigkeit so eingestellt, daß bis zum nächsten Morgen $1500 \mathrm{ml}$ „organische Fraktion" die Säulen passiert haben. Danach wird die Ionenaustauschersäule entfernt. Die Retardionsäulen werden weiter mit 10 bis 12 aqua destillata bei einer Durchflußgeschwindigkeit von 350 bis $400 \mathrm{ml} / \mathrm{h}$ durchgespült und sind am nächsten Morgen zur Aufnahme der nächsten Meerwasserprobe bereit. In der Zwischenzeit wird der Ionenaustauscher mit etwa $500 \mathrm{ml}$ aqua destillata gewaschen und dann mit etwa 1,1 1 0,3 m Piperidinlösung beziehungsweise 1 I n-Essigsäure eluiert, worauf wieder eine Waschung mit aqua destillata, die zweckmäßigerweise über Nacht durchgeführt wird, erfolgt. Anschließend wird mit $2 \mathrm{n} \mathrm{HCl}$ beziehungsweise $2 \mathrm{n} \mathrm{NaOH}$ regeneriert. Es ist zweckmäßig, pro Retardionsäulenkombination 2 Austauschersäulen bereitzuhalten, von denen die eine im Austauschbetrieb, die andere im Regenerierbetrieb steht.

Bei Verwendung von 2 Retardionsäulenkombinationen kann täglich eine Seewasserprobe verarbeitet werden. Da die Entsalzung und das Konzentrieren der Eluate nur wenige eigentliche Arbeitsschritte mit geringem Zeitaufwand erfordern, können die Analysen der organischen Stoffe bequem in der Zwischenzeit durchgeführt werden.

Es ist selbstverständlich unerläßlich, daß die Charakteristika der verwendeten Säulen (Beginn und Ende der Elution etc.) experimentell, am besten unter Verwendung eines Fraktionssammlers, untersucht werden. Die in dieser Arbeit angegebenen Daten beziehen sich nur auf die hier angewandten Säulenmaße. Durch Vermehrung der

Tabelle 2

Entsalzung mit Retardion und nachfolgende alkoholische Extraktion des eingedampften Rückstandes. Probe: 11 künstliches Meerwasser, Durchschnittswerte von jeweils 7 Versuchen

\begin{tabular}{|cccc|}
\hline Ionen & vorgelegt & $\begin{array}{c}\text { im alkoholischen } \\
\text { Extrakt gefunden }\end{array}$ & Entsalzungsgrad \\
\hline $\mathrm{Cl}^{-}$ & $19,34 \mathrm{~g}$ & $1,931 \mathrm{~g}$ & $90,02 \%$ \\
$\mathrm{SO}^{-+}$ & $2,241 \mathrm{~g}$ & $0,041 \mathrm{~g}$ & $98,18 \%$ \\
$\mathrm{Ca}^{++}$ & $0,417 \mathrm{~g}$ & $0,0013 \mathrm{~g}$ & $99,69 \%$ \\
$\mathrm{Mg}^{++}$ & $1,327 \mathrm{~g}$ & $0,060 \mathrm{~g}$ & $95,48 \%$ \\
\hline
\end{tabular}

theoretischen Platten kann möglicherweise ein noch günstigerer Trenneffekt erzielt werden. Die hier angegebenen Säulenabmessungen wurden mehr oder weniger willkürlich angewandt, sie richteten sich nach dem vorhandenen Glasmaterial.

Es wurde weiterhin versucht, die Restentsalzung auf andere Weise durchzuführen. Die „organische Fraktion " wurde im Vakuum eingeengt und mit mehreren Portionen à $25 \mathrm{ml} 80 \%$ Athanol extrahiert. Die Konzentration der Restsalze konnte dadurch weiterhin reduziert werden (Tab. 2), insbesondere wurden die Sulfationen fast quanti- 
tativ entfernt, da sie im Retardioneluat als Alkalisulfate (SCHAEFER 1964) vorliegen. Jedoch war der Entsalzungsgrad noch nicht günstig genug, so daß die Entfernung der Restionen, besonders der Chloride, wiederum mit allerdings wesentlich kleineren Ionenaustauschersäulen hätte erfolgen müssen. Damit aber wäre es zu einem umständlicheren Drei-Schritt-Verfahren gekommen.

Es wurde bereits früher nachgewiesen (SCHAEFER 1964), daß die Ionen der Erdalkali durch Retardion relativ stark gebunden werden. Zu ihrer Entfernung aus der Säule wird eine erhebliche Wassermenge benötigt. Um den Auswaschprozeß und damit die Arbeitszeit zu verkürzen, wurde versucht, die Erdalkali-Ionen nach dem Auslauf der "organischen Fraktion" mittels 0,2 $\mathrm{m}$ ADTE in $\mathrm{NH}_{3}-\mathrm{NH}_{4} \mathrm{Cl}$-Puffer in Komplexe zu überführen. Jedoch wurde nur ein Teil der Ionen komplexiert, der die Säule früher als die nicht komplexierten Ionen verließ. Die unvollständige Komplexbildung war darauf zurückzuführen, daß in der Säule allmählich eine Auftrennung von ADTE und Puffer und damit eine Verschiebung des Gleichgewichts der Reaktion auftrat. Da der Gipfel von ADTE vor dem von $\mathrm{NH}_{4}+1$ lag, müßte mit der Aufgabe des Puffers früher begonnen werden. Dadurch wäre die Komplexbildung günstiger, und der Auswaschprozeß könnte um etliche 1 Wasser verkürzt werden. Der Aufwand an Komplexbildnern und zusätzlicher Arbeitszeit ist aber doch so groß, daß die Vorteile gegenüber dem normalen Verfahren zumindest aufgehoben würden. Weitere Versuche in dieser Richtung wurden daher nicht mehr unternommen.

Mit Hilfe des Retardionverfahrens können auch Kohlenhydrate und organische Säuren aus einer größeren Salzmenge isoliert werden (Schazfer 1964). Die Kohlenhydrate werden in der "organischen Fraktion" quantitativ wiedergefunden, die Ausbeute für die meisten organischen Säuren ist aber geringer, da ihre Elution zum Teil mit der der Chloride zusammenhängt. Zur Vollentsalzung können auch hier Ionenaustauscher eingesetzt werden. Die organischen Säuren werden an Anionenaustauscher (günstig ist die Formiatform) gebunden und mit 5 n Ameisensäure eluiert. Die Kohlenhydrate erscheinen im Effluent der Austauscher.

\section{ANWENDUNGSBEISPIELE}

(1) An drei Stationen in der Nähe Helgolands wurden Wasserproben mit einem innen metallfreien Van-Dorn-Schöpfer entnommen, sofort in Plastikflaschen eingefüllt und spätestens nach 6 Stunden eingefroren oder nach spätestens $1 / 2$ Stunde (Kabeltonne) filtriert und verarbeitet. Die frischen beziehungsweise aufgetauten Proben wurden zunächst durch ein feines Perlonfilter und dann bei 400 bis 500 Torr durch ein Membranfilter $(0,25 \mu$ Porenweite) filtriert und jeweils 11 in der früher beschriebenen Weise auf die Retardionsäulen gegeben. Die „organische Fraktion" wurde durch eine 200-mlSäule Dowex $2\left(\mathrm{OH}^{-}\right)$geleitet und die Aminosäuren und ein Teil der organischen Säuren mit n Essigsäure eluiert. Das im Rotationsverdampfer mehrmals eingeengte Eluat wurde mit Wasser auf ein Volumen von $0,5 \mathrm{ml}$ gebracht und aliquote Teile papierchromatographisch ein- und zweidimensional auf Aminosäuren untersucht (Laufmittel: n-Butanol-Eisessig-Wasser $4: 1: 1$ und Pyridin-Amylalkohol-Wasser 7,5:7:6, 
Färbung mit Ninhydrin und Folin's Reagens: $\beta$-Naphthochinonsulfonat). An Hand von Testsubstanzen mit bekannten Konzentrationen wurden die Flecken identifiziert und in einigen Fällen eine vorsichtige Abschätzung der Konzentrationen vorgenommen. Zur Identifizierung der organischen Säuren wurde mit Athanol-Ammoniak-Wasser $80: 5: 15$ und n-Butanol-Ameisensäure-Wasser 10:1:5 chromatographiert und die organischen Säuren mit dem Indikatorreagens nach PASkovA \& MUNK (1960) angefärbt. Die papierchromatographische Analyse der Kohlenhydrate erfolgte eindimensional mit n-Butanol-Eisessig-Wasser $4: 1: 1$ und mit n-Butanol-Pyridin-Wasser $6: 4: 3$, Anfärbung mit Triphenyltetrazoliumchlorid und mit Perjodat-Benzidin- $\mathrm{KMnO}_{4}\left(\mathrm{H}_{\mathrm{AIS}}\right.$ \& MACEK 1958). Die Ergebnisse der Analysen der 3 Wasserproben zeigt Tabelle 3.

Tabelle 3

Gelöste organische Substanzen in Meerwasserproben aus det Deutschen Bucht (Südliche Nordsee). 11 Proben aus $0,5 \mathrm{~m}$ Tiefe

\begin{tabular}{|c|c|c|c|c|}
\hline Station & Datum & Position & Aminosäuren & $\begin{array}{l}\text { organische } \\
\text { Säuren }\end{array}$ \\
\hline $\begin{array}{l}\text { Kabeltonne } \\
\text { (zwischen Helgo- } \\
\text { land und Düne) }\end{array}$ & 6.11 .64 & $\begin{array}{c}54^{0} 11,3^{\prime} \mathrm{N} \\
7^{0} 53,9^{\prime} \mathrm{E}\end{array}$ & $\begin{array}{l}\text { Glycin/Serin } \\
\text { Histidin } \\
\text { Arginin (Spuren) } \\
2 \text { weitere Fledken, } \\
\text { rot mit Folin }\end{array}$ & negativ \\
\hline Helgolandtonne & 20. 1.65 & $\begin{array}{c}54^{0} 9,1^{\prime} \mathrm{N} \\
7053,5^{\prime} \mathrm{E}\end{array}$ & $\begin{array}{l}\text { Glutaminsäure } \\
\text { (etwa } 50 \gamma / 1) \\
\text { Alanin }(10-20 \gamma / 1) \\
\text { Glycin (etwa } 50 \% / 1) \\
\text { Arginin (Spuren) } \\
\gamma \text {-Aminobuttersäure } \\
\text { (Spuren) } \\
\text { mehrere nicht identifizierte } \\
\text { Flecken }\end{array}$ & $\begin{array}{l}\text { Ascorbinsäure } \\
1 \text { nicht identi- } \\
\text { fizierte Säure }\end{array}$ \\
\hline Tonne $\mathrm{H} 1$ & 20. 1.65 & $\begin{array}{cc}54^{0} & 3,0^{t} \mathrm{~N} \\
8^{0} & 5,0^{\prime} \mathrm{E}\end{array}$ & $\begin{array}{l}\text { Glycin } \\
\text { Histidin } \\
\text { Arginin } \\
1 \text { unbekannte Substanz }\end{array}$ & Ascorbinsäure \\
\hline
\end{tabular}

(2) Das Wasser einer Kultur von Artemia salina und einer eingeißligen kugeligen Grünanlage wurde durch ein enges Perlonfilter und dann durch ein Membranfilter $(0,25 \mu$ Porenweite) filtriert, $450 \mathrm{ml}$ des Filtrates ad 11 aqua destillata aufgefüllt und durch die Retardionsäulen passiert. Die „organische Fraktion" wurde durch $200-\mathrm{ml}$ Säulen von Dowex $50\left(\mathrm{H}^{+}-\right.$Form) und Dowex 2 (Formiatform) geleitet. Die Aminosäuren wurden mit $110,3 \mathrm{~m}$ Piperidinlösung und die organischen Säuren mit $800 \mathrm{ml}$ 5n Ameisensäure eluiert. Die Zucker erschienen im Effluent von Dowex 2. Die mit dem Rotationsverdampfer mehrmals eingeengten Eluate wurden gefriergetrocknet und auf ein Volumen von 0,5 bis $1 \mathrm{ml}$ gebracht. Die papierchromatographische Analyse erfolgte wie oben beschrieben. 
Es wurden gefunden, bezogen auf $450 \mathrm{ml}$ Kulturfiltrat:

Cystin (etwa 50-100 $\gamma$ )

Glutaminsäure (etwa $50 \gamma$ )

Arginin (Spuren)

Serin/Glycin (etwa $50 \gamma$ )

$\beta$-Alanin (Spuren)

Leucin (Spuren)

Valin (Spuren)

mehrere nicht identifizierte Substanzen:

die sich mit Ninhydrin bzw. Folin

anfärben ließen
Ascorbinsäure

eine weitere, nicht identifizierte Säure

Rhamnose (etwa $500 \%$ )

1 nicht identifizierter reduzierender

Zucker

1 nicht identifizierter, nicht reduzierender

Zucker

(3) Aus einer gealterten Enteromorpha- und Harpacticiden-Kultur wurde eine Probe entnommen, wie oben filtriert und durch 2 kleine Retardionsäulen (SCHAEFER 1964) geschickt. Eine Restentsalzung wurde nicht vorgenommen. Im Kulturmedium wurde der Gehalt an organischem C nach GrLbBricht (1957) und im Säuleneluat der Gehalt an ninhydrinpositiven Substanzen bestimmt.

Es wurden gefunden:

C-Aquivalente $\quad 17,68 \mathrm{mg} / 1$ Papierchromatographie: einige ninhydrinninhydrinpositive Substanzen, positive Substanzen, deren Identifizierung als Leucin ausgedrückt: $\quad 10,1 \mathrm{mg} / 1$ wegen der Restsalze nicht möglich war Nach zwei Tagen wurde der Kultur eine weitere Wasserprobe entnommen und wie oben behandelt. In dieser Probe wurden gefunden: C-Aquivalente 12,8 $\mathrm{mg} / 1$; ninhydrinpositive Substanzen: nil; Papierchromatographie: nil.

\section{DISKUSSION}

Mit Hilfe des Ionenverzögerungsverfahrens können einige Gruppen von organischen Substanzen weitgehend von anorganischen Salzen getrennt werden (ScHAEFER 1964). Bei der Ubertragung der früher geschilderten Versuche auf einen größeren Maßstab war jedoch der Entsalzungsgrad für die meisten Substanzen geringer, als erwartet werden mußte. Der Grund dafür ist folgender: Die in der vorhergehenden Arbeit beschriebenen Versuche wurden mit einem 1962 gekauften Retardionmaterial durchgeführt. Vorversuche mit 1963 gekauftem Retardion ergaben, daß dieses Material nicht identisch war mit der Charge von 1962. Die Trennung von Glycin und $\mathrm{NaCl}$ war bei beiden Chargen unterschiedlich (Abb. 1). Bei dem "schlechteren“ Präparat 1963 stieg die Front der Chloridionen bereits früher an. Das führte dazu, daß die in der „organischen Fraktion" enthaltene Restchloridkonzentration etwa 3-bis 4mal höher lag als bei den Versuchen mit der guten Qualität. Es war ferner auffallend, daß Farbe und Geruch der beiden untersuchten Chargen differierten. Der Grund der Unterschiede der beiden Retardionpräparate konnte noch nicht ermittelt werden, da die Kontrollnummern unbekannt waren und deshalb Rückfragen bei dem Herstellungswerk nicht beantwortet werden konnten. Versuche, durch Behandlung mit Säuren und Laugen in der Kälte und 


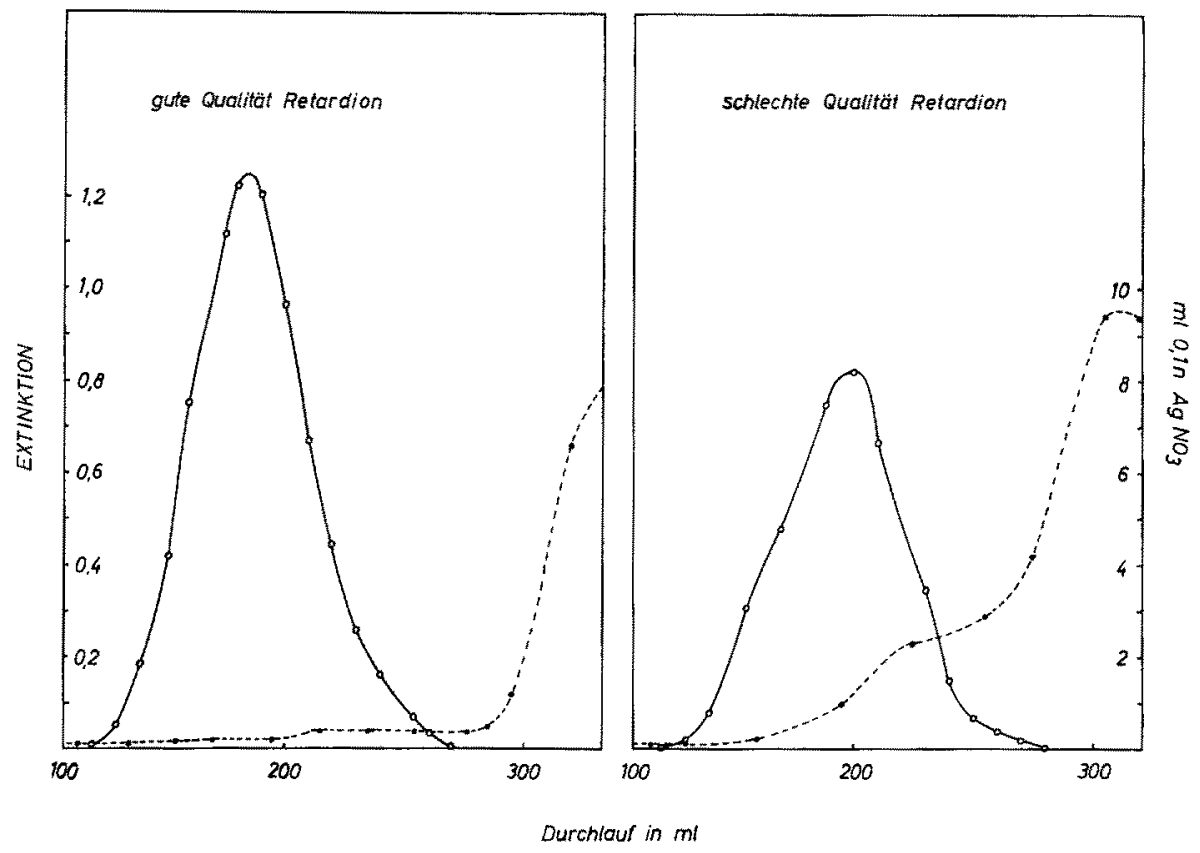

Abb. 1: Trennung von Glycin und $\mathrm{Cl}^{\prime}$ mit zwei Retardion 11A8-Chargen. 2 kleine Säulen. 1-2 mg Glycin, $50 \mathrm{ml}$ Seewasser. o—o Glycin, $\bullet-\cdots \mathrm{Cl}^{\prime}$

in der Hitze eventuelle Verunreinigungen aus dem Harz zu entfernen, erbrachten Keine besseren Trennungsergebnisse. Die Restkapazität für Anionen differierte etwas: gute Qualität $0,242 \mathrm{mval} / \mathrm{ml} \mathrm{Harz}$, schlechte Qualität $0,271 \mathrm{mval} / \mathrm{ml} \mathrm{Harz}$. Ein kürzlich zur Verfügung gestelltes weiteres Präparat von Retardion 11A8 mit der Kontrollnummer 4360-4 zeigte ebenfalls die Eigenschaften der schlechteren Qualität. Die Kurven der Abbildung 1 haben, besonders was die Front der Chloride betrift, eine große Ahnlichkeit mit denen der Abbildung 8 aus der Arbeit von Hatch et al. (1957). Dort wurden zwei Retardionpräparate miteinander verglichen, die sich im Vernetzungsgrad unterschieden. Ahnliche Unterschiede im Verlauf der Chloridfront zeigen Abbildungen 5 und 6 der Arbeit von Hatch \& SMITH (1961). Hier werden die Trennergebnisse mit Retardion $11 \mathrm{~A} 8(8 \%)$ und Retardion $51 \mathrm{~A} 2(2 \% \mathrm{DVB})$ einander gegenübergestellt. Es ist daher anzunehmen, daß die gute Qualität, wie sie in meiner früheren Arbeit verwendet wurde, dem Fabrikationstyp Retardion $11 \mathrm{~A} 8$ (8\% DVB) entspricht, während die schlechte Qualizät ein Fabrikat mit anderem Vernetzungsgrad ist.

Die in der vorliegenden Arbeit mitgeteilten Versuche wurden mit der schlechteren Qualität durchgeführt. Abbildung 2 zeigt, daß auch hier die Chloridfront deutlich ausgebildet ist und in der "organischen Fraktion" bereits stark als KCl-Gipfel ansteigt. Dadurch muß in der "organischen Fraktion" eine größere Menge Restsalze verbleiben. Allerdings erscheint die Hauptmenge der Chloride erst nach $3100 \mathrm{ml}$ Durchlauf, dieser Hauptgipfel wird in der Abbildung nicht mehr gezeigt, da er für die Diskussion unerheblich ist. Dadurch konnte auch mit der schlechteren Retardion-Qualität noch eine 


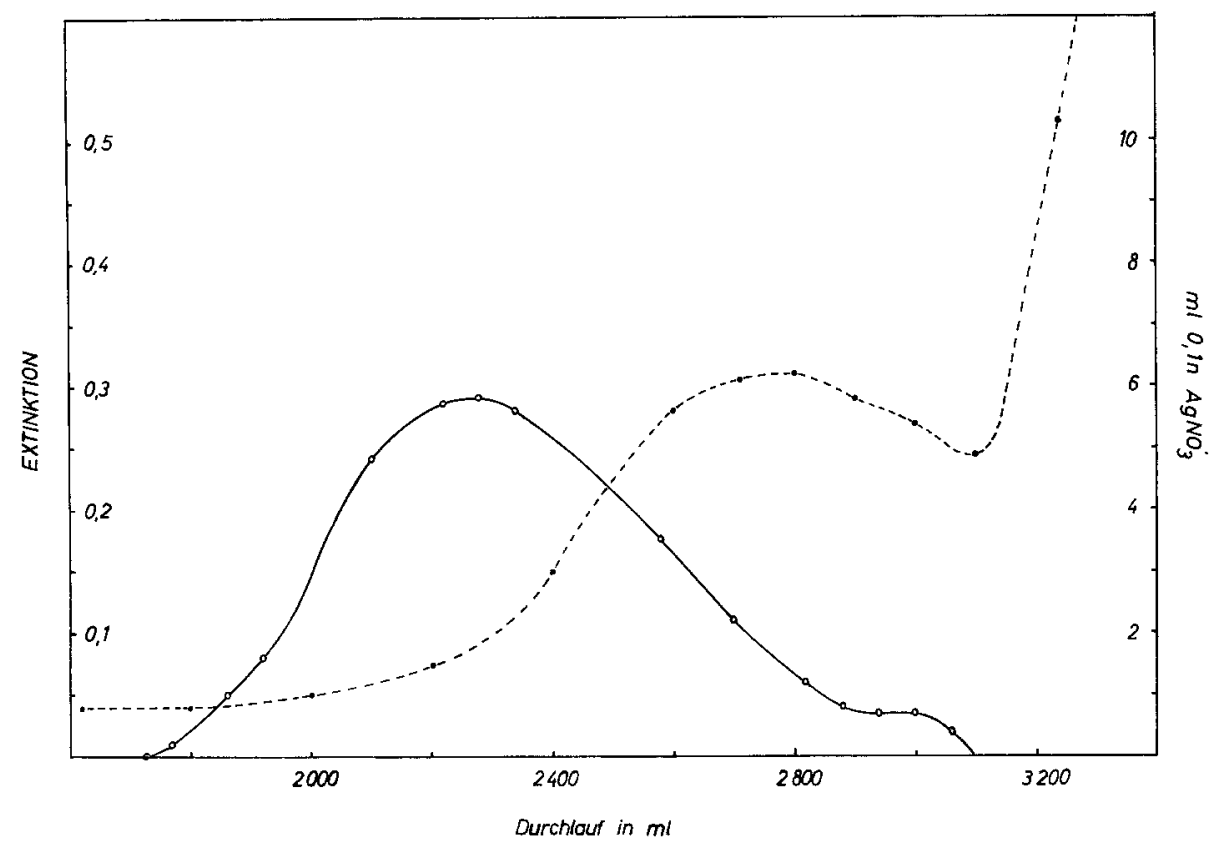

Abb. 2: Trennung von Glycin und $\mathrm{Cl}^{\prime}$ mit der Dreisäulenkombination Retardion $11 \mathrm{~A} 8.5 \mathrm{mg}$ Glycin, 11 Seewasser. o Glycin,

relativ gute Abtrennung der Aminosäuren von den Salzen erreicht werden (vgl. Tab. 1). Diese würde jedoch zweifelsohne bei Anwendung der besseren Charge, die mir damals nicht mehr zur Verfügung stand, wesentlich günstiger ausfallen, so daß dann weitere Einsparungen an Ionenaustauschern und Elutions- und Regeneriermitteln zur Restentsalzung erfolgen könnten. Es ist zu hoffen, daß der Grund der unterschiedlichen Qualitäten von Retardion von dem Herstellerwerk geklärt werden kann, und daß dann, zumindest für die Belange der Entsalzung biologischer Flüssigkeiten, die gute Qualität wieder auf dem Markt erscheint. Es muß ausdrücklich hervorgehoben werden, daß die Prüfung der verschiedenen Retardionchargen unter identischen Versuchsbedingungen erfolgte.

Durch verlängertes Nachspülen mit Wasser könnte der Entsalzungsgrad noch weiter herabgesetzt werden. Jedoch wären dazu mindestens 501 Wasser notwendig, um auf ein Minimum der Alkalichloride in der "organischen Fraktion" zu kommen. Der Restgehalt an Alkalisulfaten würde aber dadurch nur unwesentlich reduziert werden. $\mathrm{Da}$ eine so große Wassermenge und der entsprechende Zeitaufwand für laufende Untersuchungsreihen zu groß sind, wurde aus praktischen Gründen bei einer Spülmenge von 12 bis 151 Wasser verblieben und die Ionenaustauschermenge für die Restentsalzung etwas heraufgesetzt.

Da die Entsalzung mit Retardion partiell verläuft und für die weitere Analyṣe der isolierten organischen Substanzen meist eine Vollentsalzung notwendig ist, wurde die Restentsalzung durch Ionenaustauscher vorgenommen. Durch Vorversuche wurde ermittelt, in welchem Elutionsbereich Aminosäuren, Kohlenhydrate etc. die Retardion- 
säulen verließen. In diesem Bereich wurden die Retardion-mit den Austauschersäulen verbunden. Dadurch lassen sich die isolierten Verbindungen weiter in bekannter Weise auftrennen. Zur Isolierung der genannten Substanzgruppen aus dem Meerwasser bedarf es also nur eines Zweischrittrerfahrens: Hauptentsalzung durch Retardion, Restentsalzung durch Ionenaustauscher. Die Verwendung dieses Verfahrens hat gegenüber dem Einschrittverfahren: Entsalzung nur durch Ionenaustauscher, den großen Vorteil, daß sehr erhebliche Mengen an Elutions- und Regeneriermitteln sowie an Zeit eingespart werden können, was sich besonders bei Reihenuntersuchungen bemerkbar macht. Die Zahl der einzelnen Arbeitsschritte ist sehr gering, die Zeitintervalle zwischen ihnen lassen sich durch Vorversuche ermitteln. Eine Arbeitskraft kann daher eine Reihe von Säulenkombinationen gleichzeitig bedienen. Damit könnte die Retardionmethode auch für kleinere Reihenuntersuchungen Verwendung finden.

Die Anwendungsbeispiele zeigen, daß mit Hilfe des geschilderten Verfahrens Aminosäuren aus dem Meerwasser isoliert werden konnten. $\mathrm{Da}$ die Arbeiten abgebrochen werden mußten, war die quantitative Analyse nicht mehr möglich. Die Zahl der isolierten Aminosäuren ist nicht groß; mehrere Autoren (Tatsumoto et al. 1961, PARK et al. 1962, Stephens 1962, 1963, Palmork 1963a, b und Degens et al. 1964) fanden bis zu 18 Aminosäuren in den von ihnen bearbeiteten Proben, zum Teil aus anderen Meeren. In unserem Fall war die Jahreszeit der Probenentnahme sehr ungünstig, der Planktongehalt war sehr niedrig (HAGMEIER, unpubliziert). Es ist anzunehmen, daß während und nach der Planktonblüte erheblich mehr Aminosäuren in höheren Konzentrationen hätten gefunden werden können.

Die Isolierung von organischen Säuren ist durch das Ionenverzögerungsverfahren ebenfalls möglich (Schaefer 1964), jedoch ist die Ausbeute der meisten Säuren in der vorteilhaften "organischen Fraktion" nicht quantitativ. Diese Methode ist daher zunächst zur qualitativen Isolierung und Bestimmung von organischen Säuren aus dem Meerwasser geeignet. Es ist indessen möglich, durch Vorversuche zu überprüfen, wieviel Prozent von aufgegebenen Säuren in det „organischen Fraktion“ wiedergefunden werden. Da dieser Anteil nach den bisherigen Erfahrungen bei strikter Einhaltung der Versuchsbedingungen für jede Säure konstant sein müßte, wäre die Anbringung von Korrektionsfaktoren und dadurch die rechnerische Ermittlung der wirklichen Konzentration möglich.

Die Retardionmethode ist hingegen für die Isolierung von Kohlenhydraten und von etlichen organischen Phosphatverbindungen sehr geeignet. Letztere konnten in der untersuchten Probe einer Planktonkultur nicht gefunden werden. Jedoch ließen sich Rhamnose und zwei noch nicht identifizierte Zucker isolieren.

Neben einigen unbekannten organischen Säuren konnte Ascorbinsäure in der Deutschen Bucht festgestellt werden. WANGERSKY (1952) hatte bereits über die Isolierung von Ascorbinsäure (neben einem Rhamnosid) aus Meerwasser berichtet, sein Befund konnte also bestätigt werden. 


\section{ZUSAMMENFASSUNG}

1. Es wird eine kritische Übersicht über die bisherigen Methoden für die Isolierung von Aminosäuren aus dem Meerwasser gegeben.

2. Ein weiteres Isolierungsverfahren wird ausführlich beschrieben. Filtriertes Seewasser passiert 3 mit Retardion 11A8 gefüllte Säulen verschiedener Abmessungen. Dabei wird der Auslauf des größten Teils der anorganischen Ionen verzögert und so ein hoher Entsalzungsgrad der die Aminosäuren enthaltenen Fraktion (= „organische Fraktion") erreicht.

3. Die "organische Fraktion" wird anschließend durch Kat- oder Anionenaustauscher vollentsalzt. Nach Einengen des Eluates im Rotationsverdampfer können die Aminosäuren durch geeignete chromatographische Verfahren identifiziert und quantitativ bestimmt werden.

4. Mit Hilfe des geschilderten Verfahrens können auch Kohlenhydrate, organische Phosphatverbindungen und organische Säuren isoliert werden. Die Ausbeute ist aber bei den organischen Säuren nicht quantitativ.

5. Es werden einige Anwendungsbeispiele mitgeteilt. Aus mehreren Wasserproben der Deutschen Bucht sowie aus zwei Planktonkulturen wurden verschiedene Aminosäuren, Rhamnose und zwei weitere Zucker, Ascorbinsäure und zwei noch nicht identifizierte organische Säuren isoliert.

Dem Direktor der Biologischen Anstalt Helgoland, Herrn Prof. Dr. O. KInne, danke ich für einen Arbeitsplatz und für sein großes Interesse an der vorliegenden Arbeit. Besonderer Dank gebührt Herrn Dr. H. Aurich, Litoralstation der Biologischen Anstalt Helgoland (List auf Sylt), für seine Unterstützung. Herrn Dr. Grubhofer, Serva-Entwicklungslabor Heidelberg, danke ich sür Ratschläge bei der Bearbeitung des Problems der unterschiedlichen Retardionchargen. Herr Dr. E. HAGmerer, Meeresstation der Biologischen Anstalt Helgoland, besorgte mir liebenswürdigerweise die Meerwasserproben.

\section{ZITIERTE LITERATUR}

Belser, W. L., 1959. Bioassay of organic micronutrients in the sea. Proc. natn Acad. Sci. USA. 45, 1533-1542.

Buchanan, D. L., 1957. Desalting amino acid solutions by displacement with piperidine. Analyt. Chem. 29, 1877-1888.

Degens, E. T., Reuter, J. H., \& Shaw, K. N. F., 1964. Biochemical compounds in offshore California sediments and sea waters. Geochim. cosmodim. Acta 28, 45-66.

GlLlbRICHT, M., 1957. Ein Verfahren zum oxydativen Nachweis von organischer Substanz in Seewasser. Helgoländer wiss, Meeresunters. 6, 76-83.

HaIS, I. M. \& MaceK, K, 1958. Handbuch der Papierchromatographie. A. d. Tschech. Bd. 1. G. Fischer, Jena, 860 pp.

Hatch, M. J., Dillon, J. A. \& SMrth, H. B., 1957. Preparation and use of snake-cage polyelectrolytes. Ind. Engng. Chem. 49, 1812-1819.

$\mathrm{HATCH}_{3}$ M. J. \& SMrTH, H. B., 1961. Use of snake-cage polyelectrolytes for purifying glycerine. J. Am. Oil Chem. Soc. 38, 470-473.

JERREY, L. M. \& Hood, D. W., 1958. Organic matter in sea water; an evaluation of various methods for isolation. J. mar. Res. 17, 247-271.

Johnston, R., 1955. Biologically active compounds in the sea. J. mar. biol, Ass. U. K. 34, 185-195. 
Newell, B., 1962. Sorption of dissolved phosphorus from sea-water by means of aluminium hydroxide. Nature, Lond. 195, 72-73.

Palmork, K. H., 1963a. Studies of the dissolved organic compounds in the sea. FiskDir. Skr., Ser. Havundersgk. 13, 120-125.

- 1963b. The use of 2,4-Dinitro-1-fluorobenzene in the separation and identification of amino acids from sea water. Acta chem. scand, 17, 1456-1457.

Park, K., Wriliams, W. T., Prescott, J. M. \& Hood, D. W., 1962. Amino acids in deep-sea water. Science, N.Y. 138, 531-532.

Paskova, J. \& Munk, V., 1960. A combined detecting reagent for the identification of organic acids on paper chromatograms. J. Chromat. 4, 241-243.

Schaefer, H., 1964. Beiträge zur Entsalzung mit Retardion 11A8. Helgoländer wiss. Meeresunters. 11, 301-322.

- 1965. Entsalzung mit dem Ionenverzögerungsverfahren. Naturwissenschaften 52, 131.

Stephens, G. C., 1962. Amino acids in the economy of the bamboo worm, Clymenella torquata. Biol. Bull. mar. biol. Lab., Woods Hole 123, 512 .

- 1963. Uptake of organic material by aquatic invertebrates. 2. Accumulation of amino acids by the bamboo worm, Clymenella torquata. Comp. Biochem. Physiol. 10, 191-202.

Tatsumoto, M., Williams, W. T., Prescott, J. M. \& Hood, D. W., 1961. Amino acids in samples of surface sea water. J. mar. Res. 19, 89-96.

WANGERSKY, P. J., 1952. Isolation of ascorbic acid and rhamnosides from sea water. Science, N.Y., 115, 685. 